\title{
Tratamento Medicamentoso da Insuficiência Cardíaca em Hospital Terciário de São Paulo
}

\author{
A ntonio Carlos Pereira Barretto, Mauricio W ajngarten, João Batista Serro-A zul, Humberto Pierri, \\ A mit $\mathrm{N}$ ussbacher, $\mathrm{O}$ távio Celso Eluf Gebara
}

São Paulo, SP

\begin{abstract}
Objetivo - Verificar como são tratados pacientes com insuficiência cardíaca (IC) em hospital terciário de São Paulo.

Métodos - Analisados 100 pacientes com IC, em tratamento ambulatorial, com idade média de 56,8 anos, sendo 76 homens. Todos realizaram estudo ecocardiográfico, que identificou diâmetros ventriculares entre 48 e $89 \mathrm{~mm}$ (média 65,9) e fração de ejeção (FE) entre 0,22 e 0,59 (média 0,43). A etiologia da disfunção ventricular (DV) foi isquêmica em 42 casos, cardiomiopatia dilatada em 28 , secundária à valvopatia em 12, doença de Chagas em 10 e cardiomiopatia hipertensiva em 8. Analisou-se a terapêtica prescrita, se continha inibidores da enzima conversora de angiotensina (IECA) e qual a sua dosagem. Foi analisado, também, se a etiologia ou o grau de DV tinha influência na prescrição.

Resultados - Oitenta e sete pacientes receberam IECA, sendo que em 31 a dose foi inferior à preconizada nos grandes estudos. Digoxina foi prescrita em 69 casos, diuréticos em 85 e ácido acetilsalicílico em 33. Dividindo os pacientes em dois grupos, com FE maior e menor que 0,45 , observamos que, no último, foi maior a prescrição de IECA $(91,5 \%$ vs $80,4 \%)$ e maior o uso de doses adequadas (61\% vs 48,7\%).

Conclusão - A maioria dos pacientes foi tratada conforme recomendações atuais, apresentando boa tolerabilidade para IECA, contudo 1/3 deles não usou IECA em dose considerada adequada. Esquemas terapêticos com betabloqueadores e inibidores da angiotensina II não fizeram parte da prática clínica rotineira.
\end{abstract}

Palavras-chave: disfunção ventricular, insuficiência cardíaca, inibidores da enzima conversora de angiotensina

\section{Heart Failure Medical Treatment in a Tertiary Hospital of São Paulo}

Purpose - To study how patients with heart failure $(H F)$ are treated in a tertiary hospital in São Paulo.

Methods - One hundred patients with HF during ambulatory care were analyzed. Seventy-six were men, and the average population age was 56.8 years old. All patients were submitted to echocardiogram, which identified ventricular diameters ranging between 48 and $89 \mathrm{~mm}$ ( $a v$ erage 65.9) and ejection fraction (EF) between 0.22 and 0.59 (average 0.43). The cause of HF was ischemic in 42 cases, dilated cardiomyopathy in 28 , valvular heart disease in 12, Chagas' disease in 10 and systemic hypertension in 8 patients. The prescribed treatment was analyzed, with attention to the prescription and dosage of angiotensin converting enzyme (ACE) inhibitors. We also analyzed whether the cause and/or the degree of HF influenced the treatment chosen.

Results - Eighty-seven patients received ACE inhibitors, 31 received doses below those recommended in the large trials. Digoxin was prescribed in 69 cases, diuretics in 85, and aspirin in 33. When dividing the patients according to EF, the group with EF below 0.45 was prescribed more often ACE inhibitors (91.5\% vs $80.4 \%$ ) and had more often usage of adequate doses (61\% vs $48.7 \%)$.

Conclusion - In this sample the majority of the patients were treated according to modern recommendations and tolerated well ACE inhibitors, however 1/3 did not receive ACE inhibitors in the recommended doses. Treatment based on betablockers or angiotensin II inhibitors were not routinely employed.

Key-words: left ventricular systolic dysfunction, heart failure, ACE inhibitors

Arq Bras Cardiol, volume 69 (nº 6), 375-379, 1997

Instituto do Coração do Hospital das Clínicas - FMUSP

Correspondência: Antonio Carlos Pereira Barretto - Incor - Divisão de Cardiologia

Social - Av. Dr. Enéas C. Aguiar, 44 - 05403-000 - São Paulo, SP

Recebido para publicação em 4/9/97

Aceito em 8/10/97
A insuficiência cardíaca (IC) é doença muito limitante e de alta morbi-mortalidade nas suas formas avançadas ${ }^{1-3}$. A terapêutica correta representa importante mecanismo para melhora desta história tão negativa ${ }^{4,5}$. 
Desde os resultados do estudo CONSENSUS ${ }^{4}$, tem se considerado que a melhor forma de se tratar IC é através da associação de digital, diurético e inibidores da enzima conversora de angiotensina (IECA).

Embora sem consenso, há evidências que os melhores resultados são obtidos com doses semelhantes às empregadas nos grande estudos multicêntricos ${ }^{6,7}$.

Outras drogas poderão ser associadas a este esquema, ou mesmo com a substituição de alguns desses medicamentos, conforme a resposta de cada paciente. Betabloqueadores $^{8}$, amiodarona ${ }^{9}$, nitratos ${ }^{5}$, hidralazina ${ }^{5} \mathrm{e}$, mais recentemente, os inibidores da angiotensina II ${ }^{10}$ vêm sendo empregados com este objetivo.

Por outro lado, antagonistas dos canais de cálcio ${ }^{11} \mathrm{e}$ ácido acetilsalicílico ${ }^{12}$ são considerados drogas que podem piorar a falência cardíaca ou interferir no efeito dos IECA e vêm tendo sua prescrição criticada nos portadores de disfunção ventricular(DV) ou IC.

Quando iniciar e a partir de que grau de DV os IECA devem ser prescritos, são ainda pontos controversos.

Em nosso meio, como há carência de dados sobre aspectos referentes às condutas médicas nas diversas doenças, procuramos verificar como os médicos de um grande hospital terciário de São Paulo estão tratando portadores de IC de diferentes etiologias.

\section{Métodos}

No ambulatório do INCOR, em maio e junho/97, de modo aleatório, foram selecionados 100 pacientes com IC que apresentavam fração de ejeção do ventrículo esquerdo (FEVE) reduzida ao ecocardiograma $(\mathrm{ECO})(<0,60)$. Os pacientes foram selecionados do ambulatório das várias equipes, em dias sucessivos, sendo desprezados os casos de $1^{\text {a }}$ e $2^{\mathrm{a}}$ consultas, a fim de evitar o início de tratamento.

Os pacientes apresentavam idades que variaram de 22

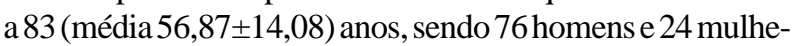
res. Ao ECO, o diâmetro do ventrículo esquerdo variou de 48 a 89 (média 65,98 77,91)mm e a fração de ejeção (FE) de 0,22 a0,59 (média0,43 00,09 ).

A etiologia da DV foi: cardiomiopatia isquêmica em 42 casos, cardiomiopatia dilatada em 28 , valvopatia em 12 , do-

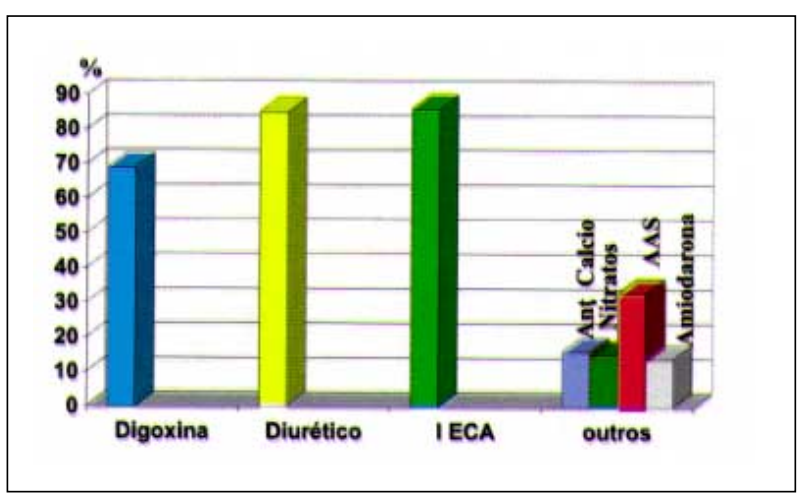

Fig. 1 - Gráfico de barras mostrando a freqüência de prescrição dos medicamentos empregados no tratamento de pacientes com insuficiência cardíaca.

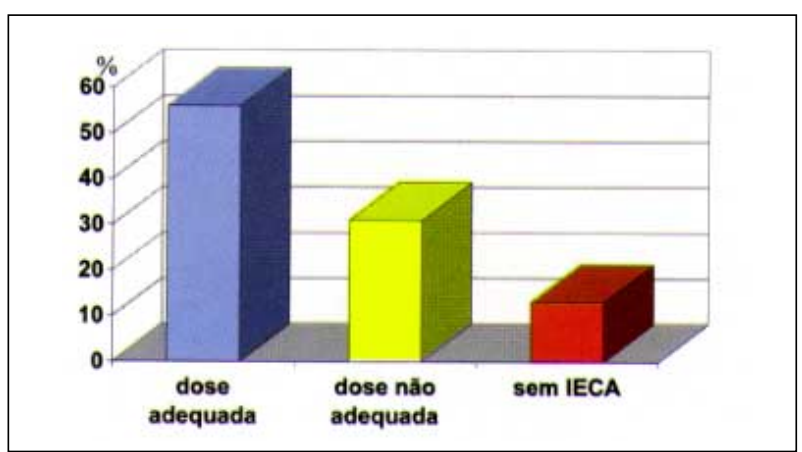

Fig. 2- Gráfico de barras mostrando frequiência de pacientes que não recebeu prescrição de IECA e daqueles com doses consideradas adequadas e não adequadas.

ença de Chagas em 10 e cardiomiopatia hipertensiva em 8.

Analisou-se se a terapêutica diferia conforme a cardiopatia de base e intensidade da DV.

Nesta casuística procurou-se verificar, na terapêutica prescrita, se estavam sendo receitados IECA e, se prescritos, em que dosagem. Consideraram-se doses diárias adequadas, doses pelo menos iguais a $75 \mathrm{mg}$ de captopril, $20 \mathrm{mg}$ de enalapril e $10 \mathrm{mg}$ de ramipril.

Análise estatística constituiu no cálculo da média e desvio-padrão para as variáveis numéricas, cálculo da freqüência e teste de Fisher para verificar se havia diferenças relacionadas às diferentes etiologias e ao grau de DV. O nível de significância utilizado foi de $5 \%$.

\section{Resultados}

Análise global da população mostrou que os pacientes com doença de Chagas são mais jovens e os com cardiomiopatia isquêmica os mais idosos (tab. I).

Considerando as diversas etiologias, observamos que cardiomiopatia dilatada é mais freqüente entre os com FE $<0,45$ e valvopatias entre os com $F E>0,45$ ( $p=0,0005)$ (tab. II).

Na população estudada, a terapêutica prescrita foi: digoxina para $69 \%$ dos pacientes, diuréticos para $85 \%$, IECA para $87 \%$, antagonista do cálcio para $16 \%$, nitratos para $15 \%$, ácido acetilsalicílico para $33 \%$, amiodarona para $14 \%$, hidralazina para $3 \%$, betabloqueador para $4 \%$ e anticoagulantes para $10 \%$ dos pacientes analisados (fig. 1). Todos tinham prescrição de mais de um medicamento.

Verificamos que o digital ( $\mathrm{p}=0,0004)$ foi mais prescrito para os pacientes com cardiomiopatia dilatada, cardiomiopatia hipertensiva e doença de Chagas, enquanto o ácido acetilsalicílico ( $\mathrm{p}>0,0001)$, os antagonistas do cálcio $(\mathrm{p}=0,049)$ e os nitrato $(\mathrm{p}=0,0053)$ foram para os pacientes com cardiomiopatia isquêmica (tab. I).

Os IECA empregados e as respectivas doses médias prescritas foram: captopril, 71,9mg/dia, enalapril, $23,7 \mathrm{mg} / \mathrm{dia}$ e ramipril, 5,8mg/dia. Observou-se também que $13 \%$ dos pacientes não receberam a medicação, $31 \%$ tiveram prescrição em doses consideradas inferiores às ideais e $56 \%$ receberam a medicação na dosagem correta (fig. 2).

A terapêutica apresentou diferença conforme a causa da DV e de acordo com o grau de comprometimento miocárdio. 


\begin{tabular}{|c|c|c|c|c|c|c|c|c|c|}
\hline & Idade & Digoxina & Diurético & IECA & AAS & Amiodarona & $\begin{array}{l}\text { Antagonista } \\
\text { do cálcio }\end{array}$ & Anticoagulantes & Nitrato \\
\hline Isquêmica & 63,0 & $20-47,6 \%$ & $31-73,8 \%$ & $35-79,5 \%$ & $25-56,8 \%$ & $5-11,3 \%$ & $12-27,2 \%$ & $2-4,5 \%$ & $13-29,5 \%$ \\
\hline Dilatada & 54,4 & $26-52,8 \%$ & $27-96,4$ & $27-96,4 \%$ & $4-14,2 \%$ & $5-17,8 \%$ & $1-3,5 \%$ & $3-10,7 \%$ & $0-0 \%$ \\
\hline Doença de Chagas & 45,7 & $8-80,0 \%$ & $9-90,0 \%$ & $9-90,0 \%$ & $1-10 \%$ & $3-30 \%$ & $0-0 \%$ & $2-20 \%$ & $0-0 \%$ \\
\hline Valvopatia & 50,0 & $8-66,6 \%$ & $10-83,3 \%$ & $10-83,3 \%$ & $3-25 \%$ & $1-8,3 \%$ & $2-16,6 \%$ & $3-25 \%$ & $1-8,3 \%$ \\
\hline Hipertensiva & 57,3 & $7-82,5 \%$ & $8-100 \%$ & $7-87,5 \%$ & $0-0 \%$ & $0-0 \%$ & $1-12,5 \%$ & $0-0 \%$ & $1-12,5 \%$ \\
\hline
\end{tabular}

A freqüência de pacientes com prescrição de doses adequadas dos IECA conforme a etiologia foi de $87,5 \%$ nos pacientes com cardiomiopatia hipertensiva, $60 \%$ nos com doença de Chagas, 59,5\% nos com cardiomiopatia isquêmica, $53,5 \%$ nos com cardiomiopatia dilatada e $33,3 \%$ nos com valvopatias $(\mathrm{p}=0,08)$.

Dividindo os pacientes em dois grupos, conforme a FE $\leq 0,45$ ou $>0,45$, verificamos diferenças na prescrição das drogas (tab. III). Assim nos pacientes com menores valores de FE houve tendência a ser maior a prescrição de doses adequadas de IECA $(\mathrm{p}=0,08)$. Betabloqueadores e antagonistas de cálcio foram mais freqüentemente prescritos para pacientes com FE >0,45 (menor comprometimento miocárdico).

\begin{tabular}{|c|c|c|}
\hline & Fração de ejeção $\leq 0,45$ & Fração de ejeção $>0,45$ \\
\hline Isquêmica & $22-52,3 \%$ & $20-47,6 \%$ \\
\hline Dilatada & $23-82,1 \%$ & $5-17,8 \%$ \\
\hline Doença de chagas & $6-60 \%$ & $4-40 \%$ \\
\hline Valvopatia & $3-25 \%$ & $9-75 \%$ \\
\hline Hipertensiva & $5-62,5 \%$ & $3-37,5 \%$ \\
\hline
\end{tabular}

\begin{tabular}{|c|c|c|}
\hline \multicolumn{3}{|c|}{$\begin{array}{l}\text { Tabela III - Número de pacientes com prescrição de cada medicamen } \\
\text { to, conforme o grau de disfunção ventricular }\end{array}$} \\
\hline & $\begin{array}{l}\text { Fração de Ejeção } \\
\quad \leqslant 0,45\end{array}$ & $\begin{array}{c}\text { Fração de Ejeção } \\
\quad>0,45\end{array}$ \\
\hline $\mathrm{n}$ & 59 & 41 \\
\hline Digoxina & $50-84,7 \%$ & $19-46,3 \%$ \\
\hline Diurético & $56-94,9 \%$ & $29-70,7 \%$ \\
\hline IECA & $54-91,5 \%$ & $33-80,4 \%$ \\
\hline Dose adequada & $36-61,0 \%$ & $20-48,7 \%$ \\
\hline Dose não adequada & $18-30,5 \%$ & $13-31,7 \%$ \\
\hline Ácido acetilsalicílico & $13-22,0 \%$ & $20-48,7 \%$ \\
\hline Amiodarona & $12-20,3 \%$ & $2-4,8 \%$ \\
\hline Antagonista do cálcio & $6-10,1 \%$ & $10-24,3 \%$ \\
\hline Anticoagulante & $5-8,4 \%$ & $4-9,7 \%$ \\
\hline Betabloqueador & $0-0 \%$ & $4-9,7 \%$ \\
\hline Hidralazina & $2-3,3 \%$ & $1-2,4 \%$ \\
\hline Nitrato & $6-10,1 \%$ & $5-21,9 \%$ \\
\hline
\end{tabular}

\section{Discussão}

$\mathrm{Na}$ IC, o tratamento clínico influencia, de maneira importante, a evolução e o prognóstico dos pacientes ${ }^{13,14}$. Desde os anos 80, com os resultados dos estudos multicêntricos ${ }^{4,5}$, a terapêutica vem sofrendo modificações, com a demonstração de que as drogas, além do efeito hemodinâmico, podem influir na sobrevida. De fato, identificou-se que a maioria dos inotrópicos, quando prescrita de maneira crônica, provoca aumento da mortalidade ${ }^{15} \mathrm{e}$ os IECA a reduzem $^{4,16}$.

Os antagonistas dos canais de cálcio vêm sendo objeto de estudo quanto aos seus efeitos em portadores de falência cardíaca. Os antagonistas de primeira geração, nifedipina, diltiazem e verapamil parecem ser deletérios à função cardíaca e podem acentuar as manifestações clínicas. Os antagonistas mais recentes como amlodipina e felodipina parecem não modificar a sobrevida dos pacientes ${ }^{12}$.

Os betabloqueadores através de seus efeitos antiadrenérgicos, considerando que a estimulação adrenérgica é deletéria aos cardíacos, vêm sendo cada vem mais estudados no controle dos quadros de falência cardíaca, apesar de seu potencial efeito inotrópico negativo ${ }^{8}$.

Nitratos e hidralazina, desde o estudo $\mathrm{VHeFT}^{5}$, são importantes opções terapêuticas, especialmente naqueles que não toleram os IECA. Inibidores da angiotensina II após o resultado do estudo ELITE ${ }^{10}$ são a mais nova opção para o tratamento da DV.

Ácido acetilsalicílico, droga de valor plenamente documentada em pacientes com coronariopatia ${ }^{12}$, parece reduzir os benefícios que os IECA poderiam induzir aos pacientes com IC, pela interferência com as prostaglandinas, que seriam estimuladas pelo aumento dos níveis de bradicinina.

Portanto, o manuseio farmacológico dos pacientes com IC vem sofrendo modificações em função dos conhecimentos recentes. Conhecimentos que, novos e antigos, são importantes no momento da escolha do melhor esquema terapêutico para o paciente com IC. No Brasil, contudo, há carência de dados sobre este manuseio.

Neste artigo, apresentamos o levantamento realizado em um grande hospital de referência do Estado de São Paulo, e como o tratamento da IC vem sendo realizado por médicos de seus vários serviços.

Foi critério de inclusão no estudo, episódio de IC com 
a presença de DV sistólica caracterizada por redução da FEVE, analisado peloECO (normal acima de 0,65). Identificamos em dias subseqüentes, nos ambulatórios do hospital, os pacientes que apresentavam DV e analisamos a terapêutica prescrita. Foram desprezados os casos de $1^{\mathrm{a}} \mathrm{e} 2^{\mathrm{a}}$ consultas, procurando-se assim evitar influência de terapêutica inicial.

Pudemos verificar que a maioria dos pacientes recebeu, conforme o esperado, o esquema considerado clássico, composto de digital, diuréticos e IECA, sendo este último o grupo prescrito com maior freqüência.

A etiologia da DV e a sua intensidade parecem influenciar a prescrição destas drogas. Assim, o digital é menos prescrito nos portadores de cardiomiopatia isquêmica e os IECA foram mais prescritos para aqueles com cardiomiopatia hipertensiva, cardiomiopatia dilatada e na doença de Chagas, em concordância com as evidências de que o digital pode não ser benéfico em pacientes com isquemia miocárdica ${ }^{18}$ e que os IECA modificam a história natural nestas cardiopatias ${ }^{4,6,16}$.

Pudemos verificar que a maioria dos pacientes com DV foi medicada com IECA, prescritos em doses consideradas adequadas $(64,7 \%)$.

Os presentes dados sugerem várias dúvidas e recomendações apresentadas na literatura. Assim, dúvidas sobre o valor da terapêutica com IECA, para pacientes com DV com FE pouco rebaixada, podem explicar porque a prescrição dos IECA foi menos freqüente nestes pacientes em relação àqueles com $\mathrm{FE}$ bem reduzida.

O mesmo raciocínio pode ser aplicado aos 10 pacientes que ficaram sem qualquer tratamento, já que oito deles tinham $\mathrm{FE}>0,45$.

O emprego de antagonistas dos canais de cálcio, raro em pacientes com DV acentuada, é mais freqüente nos portadores de cardiomiopatia isquêmica ou cardiomiopatia hipertensiva, obedecendo as tendências atuais.

Betabloqueadores foram pouco prescritos nesta população e somente receitados para pacientes com pequeno comprometimento ventricular. Amiodarona, de indicação maior para controle de arritmias ventriculares, foi mais receitada àqueles com comprometimento ventricular mais acentuado, provavelmente como reflexo da maior incidência de arritmia nesses casos.

Ácido acetilsalicílico foi empregado com relativa freqüência nesta casuística (33\%), especialmente entre coronariopatias e valvopatias, onde sua indicação não é muito discutida pelos benefícios amplamente demonstrados. Sua prescrição, entretanto, menos freqüente nos pacientes com função ventricular mais comprometida, deve-se possivelmente às suas propriedades de reduzir os benefícios dos IECA.

Dentre os pacientes sem prescrição de IECA, dois usaram hidralazina e um losartan, sugerindo, que à seme- lhança do observado em outros estudos, o uso alternativo diante da infreqüente intolerância aos IECA.

A tendência ao uso menos freqüente de IECA entre os valvopatas (33\%), provavelmente decorre da maior prioridade em corrigir a valvopatia em detrimento ao tratamento da DV que foi, ademais, na maioria deles $(75 \%)$ de pequena magnitude $(\mathrm{FE}>0,45)$.

A dose em que os IECA deveriam ser ministrados representa aspecto polêmico ${ }^{6,7}$. Embora não haja ainda comprovação de que pequenas doses não sejam eficazes, há consenso em que se deva prescrever doses semelhantes às utilizadas nos grandes estudos multicêntricos: 100 a 150mg de captopril $^{19}, 20$ a $40 \mathrm{mg}$ de enalapril $^{4,16}$, etc. Estudos comparativos sobre o emprego de doses baixas e plenas documentam o maior benefício das doses plenas ${ }^{6,7,20}$, induzindo modulação da elevada estimulação neuro-humoral, com redução do número de internações e mortalidade.

Podemos observar, entretanto, que $1 / 3$ dos casos não receberam prescrição das doses consideradas adequadas. Em alguns casos, tal dose poderia não ter sido atingida devido à presença de hipotensão arterial, mas, possivelmente, na sua maioria por estarem os pacientes compensados, corroborando com o achado de que quanto mais acentuada a DV maior o percentual dos pacientes com doses adequadas da medicação (fig. 2). Este fato indica que doses adequadas dos IECA são bem toleradas pelos pacientes mais graves.

Apesar de considerarmos que estes resultados poderiam ser melhores, foram superiores aos observados em Londres, no Hospital St George's em 1994, pois entre as prescrições para os doentes internados com diagnóstico primário de IC, $25,7 \%$ não incluíam IECA e somente $46 \%$ os incluíam em doses adequadas ${ }^{21}$.

A questão de dose foi também analisada na Califórnia, através de um questionário, e mostrou que os cardiologistas, quando comparados a médicos generalistas ou internistas, receitam mais IECA e procuram atingir as doses consideradas adequadas com mais freqüência (35\% vs $3 \%$ vs $22 \%)^{22}$.

A tolerabilidade dos IECA nessas doses ainda não foi bem documentada em nosso meio. Portanto, do ponto de vista prático, os presentes resultados representam um estímulo ao emprego de doses adequadas freqüentemente evitadas por receio.

Em conclusão, nesta amostra a maioria dos pacientes foi tratada conforme recomendações atuais e apresentou boa tolerabilidade para IECA, contudo 1/3 deles não empregou IECA em dose considerada adequada. Esquemas terapêuticos com betabloqueadores e inibidores da angiotensina II não fizeram parte da prática clínica rotineira.

Novos estudos abrangendo outras amostras populacionais devem ser realizados a fim de permitir uma generalização destas conclusões. 


\section{Referências}

1. Cowie MR, Mosterd A, Wood DA et al - The epidemiology of heart failure. Eur Heart J 1997; 18: 208-25.

2. McKee PA, Castelli WP, McNamara PM, Kannel NB - The natural history of congestive heart failure. The Framinghan Study. N Engl J Med 1971; 285: 1441-6.

3. Bocchi EA - Situação atual das indicações e resultados do tratamento cirúrgico da insuficiência cardíaca. Arq Bras Cardiol 1994; 63: 624-30.

4. The CONSENSUS Trail Study Group - Effect of enalapril on mortality in severe congestive heart failure: results of the Cooperative North Scandinavian Enalapril Survival Study (CONSENSUS). N Engl J Med 1987; 316: 1429-35.

5. Cohn JN, Archibald DG, Ziroche S et al - Effect of vasodilatador therapy on mortality in chronic congestive heart failure. Results of a Veterans Administration Cooperative Study (VHeFT). N Engl J Med 1986; 314: 1547-52.

6. Cleland JGF, Poole-Wilson PA - ACE inhibitors for heart failure: a question of dose. Br Heart J 1994; 72(suppl.): 106-10.

7. Pacher R, Globits S, Bligher-Klein Jr et al - Clinical and neurohumoral response of patients with severe congestive heart failure treated with two different captopril dosages. Eur Heart J 1993; 14: 273-8.

8. Cleland JGF, Bristow MR, Erdmann E et al - Beta-blocking agents in heart failure. Should they be used and how? Eur Heart J 1996; 17: 1629-39.

9. Dowal HC, Nul DR, Grancelli HO et al - Randomized trial of low-dose amiodarone in severe heart failure. Lancet 1994; 355: 493-8.

10. Pitt B, Segal R, Martinez FA et al - Randomized trial of losartan versus captopril in patients over 65 with heart failure (Evaluation of Losartan in the Elderly Study, ELITE). Lancet 1997; 349: 747-52

11. Furberg CD, Psaty BM, Meyer JV - Nifedipine. Doses-related increase in mortality in patients with coronary heart disease. Circulation 1995; 92: 1326-31.

12. Cleland JGT, Bulpitt CJ, Falk RH et al - Is aspirin safe for patients with heart failure? Br Heart J 1995; 74: 215-19.
13. Stevenson WG, Stevenson LW, Middlekauff HR et al - Improving survival for patients with advanced heart failure: A study of 737 consecutive patients. J Am Coll Cardiol 1995; 26: 1417-23.

14. Stevenson WG, Stevenson LW, Middlekauff HR et al - Improving survival for patients with atrial fibrilation and advanced heart failure. J Am Coll Cardiol 1996; 281: 1458-63.

15. Packer M - The search for the ideal positive inotropic agent. NEngl J Med 1993; 329: 201-2.

16. The SOLVD Investigators - Effect of enalapril on survival in patients with reduced left ventricular ejection fraction and congestive heart failure. NEngl J Med 1991; 325: 293-302.

17. Packer M, O'Connor CM, Ghalli JR et al - Effect of amlodipine on morbidity and mortality in severe chronic heart failure. N Engl J Med 1996; 335: 1107 14

18. Benerjee AK, Campbell RWF - Digoxin therapy and survival in heart failure in sinus rhythm. Int JH Cardiology 1996; 65: 9-13.

19. Pfeffer MA, Braunwald E, Moye LA et al - Effect of captopril on mortality and morbidity in patients with left ventricular dysfunction after myocardial infarction. Results on the survival and ventricular enlargement trial (SAVE) N Engl J Med 1992; 327: 669-77.

20. Packer R, Staneck B, Berger E et al - Low vs high dose in severe heart failure. Circulation 1995; 92(suppl 1): 667

21. Missouris CG, MacGregor GA - The use of angiotensin-converting enzyme in hibitors inthe treatment of heart failure in hospital practive. Posgrad Med J 1997; 73: 409-11.

22. Edep ME, Shah NB, Tateo IM, Massie BM - Differences between primary care physicians and cardiologists in management of congestive heart failure: Relation to practice guidelines. J Am Coll Cardiol 1997; 30: 518-26. 